\title{
Obtaining Mycobacteriophages Active in Concerning Atypical Mycobacteria
}

\author{
Tokseiit A. Yelekeyev ${ }^{1}$, Nazym S. Syrym², Bolat A. Yespembetov ${ }^{2}$ \\ ${ }^{1}$ Kyrgyz Research Institute of Veterinary named after A. Duysheev, Bishkek, Kyrgyzstan \\ ${ }^{2}$ Research Institute for Biological Safety Problems, Gvardeyskiy, Republic of Kazakhstan \\ Email: nazym-syrym@rambler.ru
}

How to cite this paper: Yelekeyev, T.A., Syrym, N.S. and Yespembetov, B.A. (2018) Obtaining Mycobacteriophages Active in Concerning Atypical Mycobacteria. Journal of Biosciences and Medicines, 6, 52-56. https://doi.org/10.4236/jbm.2018.65007

Received: April 24, 2018

Accepted: May 18, 2018

Published: May 21, 2018

\begin{abstract}
Background: The article outlines the ways of preparing and sowing various samples for research with a view to isolating mycobacteriophages (MBphages), as well as developing methods for their isolation. Methods: Experimental studies on the obtaining of MB phages active against tubercle mycobacteria were conducted using samples collected from environmental objects and biological material from the different areas of the country. As an indicator of test-cultures, we have used the following M. kansasii, M. avium, M. scrofulaseum, M. phlei, M. terrae, M. intracellulare, M. smegmatis. For the cultivation of Mycobacteria and MB phages were used the following nutrient media of the company HiMedia: Dubos Broth Base, Dubos Oleic Agar Base, Kirschner Medium Base-Modified, Middlebrook 7H10 Agar Base, Peizer TB Medium Base, Lowenstein Jensen medium basе и Sauton's Fluid Medium. Results: During the research, the following results were obtained: An effective method for obtaining $\mathrm{MB}$ phages from environmental objects was selected. Four types of Mbphage were obtained, lysing atypical mycobacteria from environmental objects delivered from various areas of the Republic of Kazakhstan. Conclusion: Obtained data in the development of the method of obtaining MBphage, allows us to consider it necessary to further study their biological features, as well as the possibility of using them to develop an anti-tuberculosis effective agent.
\end{abstract}

\section{Keywords}

Mycobacterium, Mycobacteriophage, MBphage, Test-Cultures, Selection, Phagolysate, The Objects of the Environment

\section{Introduction}

One of the factors hampering the development of animal husbandry is the infec- 
tious diseases of animals, among which tuberculosis occupies a special place. The problem of elimination and prevention of the disease remains relevant in connection with the wide spread of nonspecific tuberculin reactions, the presence of latent (latent) forms of infection, and cases of repeated outbreaks of infection in previously healthy facilities [1] [2] [3] [4].

The tasks of further development of animal husbandry, the increase in the number of animals and a sharp increase in their productivity, especially in the conditions of the organization of large farms on an industrial basis, require veterinary specialists and scientific workers to use all possible methods and means to ensure the veterinary welfare of such farms. The timely prevention of tuberculosis of cattle will allow to obtain livestock products of high sanitary quality and protect people from contracting this disease.

Therefore, the search for alternative methods for the prevention of tuberculosis infection, such as the use of bacteriophages, are innovative [5]-[13].

In connection with this, the aim of the study was the need to obtain mycobacteriophages in the future to create an effective anti-tuberculosis drug

\section{Methodology}

The studies were conducted at the laboratory of microbiology of the RIBSP and in some farms of the republic conditionally well with TB. All research works were carried out according to officially regulated Veterinary legislation of the Republic of Kazakhstan: "Manual on the diagnosis of TB in animals" and "Guidelines for laboratory testing of TB in animals" [14] [15].

As an indicator of test-cultures, we have used the following Mycobacterium strains-M. kansasii, M. avium, M. scrofulaseum, M. phlei, M. terrae, M. intracellulare, M. smegmatis. Isolated in different years from environmental objects.

For the cultivation of Mycobacteria and MB phages were used the following nutrient media of the company HiMedia: Dubos Broth Base, Dubos Oleic Agar Base, Kirschner Medium Base-Modified, Middlebrook 7H10 Agar Base, Peizer ТВ Medium Base, Lowenstein Jensen medium base и Sauton's Fluid Medium.

At the beginning of the experiment, we have selected and refreshed indicator $\mathrm{MB}$ test-cultures to enrich and verify the specificity of lytic action during MB phage isolation.

Sampling was carried out in different regions (South, Southeast, North and West) of the Republic of Kazakhstan.

\section{Result}

At the beginning of the experiment, we selected and refreshed indicator test cultures of mycobacteria to enrich and verify the specificity of the lytic action in the isolation of MBphages and design experiments for the selection of optimal schemes for the initial isolation of Mbphage. At the same time, they were based on the creation of conditions that ensure the phage lysis of Mycobacterium tuberculosis. Two methods were developed and applied to isolate MBphages. 
In the future, we conducted experiments to select the optimal scheme for the isolation of MB phages. This was based on the creation of conditions for the phage lysis of Mycobacterium

Mycobacterium studied to be used as a test culture in in vitro experiments during study MB.

MB test-cultures are of great importance in the isolation and study of mycobacterium phages. Therefore, in the first experience a lot of attention was paid to full biological control of mycobacteria cultures collection for confirming the source of typical properties. For this purpose, it was used culture method, the biological sample and the polymerase chain reaction (PCR).

First method. To detect Mycobacterium phages in filtrates under study it was used the method of surface plating on Petri dishes: on Petri dishes with a culture medium dried was applied $0.1 \mathrm{~cm}^{3}$ of indicator MB test-cultures in the logarithmic growth phase, the surface was triturated with a glass spatula and allowed to 10 - 15 minutes for absorbing moisture in the agar. Then the test sample was dripped on the plate and allowed to stand for 15 - 30 minutes at a room temperature. Further plates were inverted and incubated until appearance of lysis zone on the test sample application site.

Second method. On the surface of different agar media Dubos Oleic Agar Base, Middlebrook Agar Base, Peizer TB Medium Base and Lowenstein Jensen in Petri dishes in the middle was applied $100 \mathrm{ul}$ of MB test-cultures, then on the edges was inserted $900 \mathrm{ul}$ of cooled to $45^{\circ} \mathrm{C}$ semi-fluid DIR medium to protect the culture from the burning. After sowing, the Petri dish should be mixed thoroughly by gentle rotational jiggle until complete resorption of $\mathrm{MB}$ cultures for obtaining lawn growth, and then we wait for $1-2$ minutes until semi-liquid nutrient medium DIR get cold. The Petri dish on the bottom was divided into an equal distance from each other on the conventional 9 sectors, and each zone was added $10 \mathrm{ul}$ of test phages.

As a result, the presence of a phage in a particular substrate was recognized by lysis of sensitive to them microbial cultures (test culture).

Thus, our method for the isolation and titration of phages showed the best results.

Our further investigations were carried out in the direction of selection of phage clones.

For the selection of phage clones, a piece of agar taken from lysis zone was put into a test tube with $3 \mathrm{~cm}^{3}$ of liquid nutrient medium Dubos Broth Base with further adding of $0.3 \mathrm{~cm}^{3}$ of chloroform. After it was stirred thoroughly and left for a certain time at a temperature $(4 \pm 2)^{\circ} \mathrm{C}$ for total outlet of phage particles from agar. Further a drop of suspension from the tube was applied to a freshly prepared lawn of indicator culture and incubated until lysis zone on the test sample application site. In case of a negative (transparent) zone again, we believed that inhibition of culture growth caused by the action of MB. The resulting phage lysate re-examined by agar layers. 
As a result, the presence of a phage in one or another substrate was judged by lysis of a microbial test culture sensitive to it. The specificity of the isolated phages for various species of mycobacteria has been studied by their lytic ability by applying one drop of phagolysate to the freshly prepared lawns of the cultures tested, the results shown in below.

It is visually evident that our second improved method for the isolation and titration of MBphages was successful and showed positive results in the production of mycobacteriophages.

All the isolated $4 \mathrm{MB}$ phages had a titer between 107 - 109 by Appelman and 109 - 1010 by Grazia, had pronounced specificity for M. phlei, M. scrofulaseum and did not show activity against other types of mycobacterium tuberculosis.

All these phages retained their lytic activity for 2 months, were resistant to heating at $50^{\circ} \mathrm{C}$ to $70^{\circ} \mathrm{C}$ for 30 minutes. The MBphages were resistant to the action of a $10 \%$ solution of chloroform for 45 minutes.

Experiments on the isolation of MBphages of mycobacterium tuberculosis active against virulent cultures continue.

The method developed by us made it possible to isolate for the first time - 4 MBphages, lysing atypical mycobacteria from objects of the external environment delivered from various regions of the Republic of Kazakhstan.

\section{Discussion}

To identify the phages of MB in the filtrates, we used several methods. Finally, an improved method for isolating and titrating MB-phages was developed, which showed the best results.

For identification of MB phages in filtrates we have several methods. Finally, it was developed an improved method for isolation and titration MB phages, which showed the best results.

During the research the following results were obtained:

An effective method for obtaining MB phages from environmental objects was selected.

Four types of Mbphages were obtained, lysing atypical mycobacteria from environmental objects delivered from various areas of the Republic of Kazakhstan.

Obtained data in the development of the method for obtaining MBphages, allows us to consider it necessary to further study their biological features, as well as the possibility of using them to develop an anti-tuberculosis effective agent.

\section{References}

[1] Syrym, N.S., Yelekeyev, T.A., et al. (2010) Perfection of Diagnostic Methods for Tuberculosis of Cattle. Proceedings IV Int. Scientific. Conf. Young Scientists "New Directions of Development of Agrarian Science in the Works of Young Scientists", Novosibirsk, pos. Krasnoobsk, Part Two, 128-131.

[2] Basybekov, S.Z., Bazarbayev, M.B., Yespembetov, B.A., Yelekeyev, T.A., Akmatova, E.K. and Syrym, N.S. (2018) Diagnostics of Tuberculosis and Differentiation of Nonspecific Tuberculin Reactions in Animals. Brazilian Journal of Microbiology, 
49, 329-335. https://doi.org/10.1016/j.bjm.2017.07.004

[3] Bazarbaev, M.B., Syrym, N.S., Yespembetov, B.A., et al. (2017) Sensitins for Differentiating Nonspecific Reactions to PPD Tuberculin Mammalian in Cattle. The Journal of Animal \& Plant Sciences, 27, 1534-1542.

[4] Broxmeyer, L., Sosnowska, D., Miltner, E., Chacon, O., Wagner, D., et al. (2002) Killing of Mycobacterium avium and Mycobacterium tuberculosis by a Mycobacteriophage Delivered by a Nonvirulent Mycobacterium: A Model for Phage Therapy of Intracellular Bacterial Pathogens. JInfect Dis, 186, 1155-1160. https://doi.org/10.1086/343812

[5] Kutter, E. (1997) Phage Therapy: Bacteriophages as Antibiotics. Evergreen State College, Olympia, WA.

[6] Syrym, N. and Yespembetov, B. The Use of Biological Control Cultures of Mycobacteria for Use in the Study of Mycobacteriophages. Bulletin of Ulyanovsk State Agriculture Academy, 1, 77-82.

[7] Yespembetov, B.A. and Syrym, N.S. (2017) Selection of Nutrient Medium for Mycobacteriophages Isolation. In: Abstract Book of the International Conference "Centennial Celebration of Bacteriophage Research", Pasteur Institute (France), Eliava Institute of Bacteriophages, Microbiology and Virology and Tbilisi State University, Tbilisi, Georgia, 26-29 June 2017, 119.

[8] Syrym, N.S. and Yespembetov, B.A. (2017) Isolation of Mycobacteriophages from Biological Material. In: Abstract Book of the International Conference "Centennial Celebration of Bacteriophage Research”, Pasteur Institute (France), Eliava Institute of Bacteriophages, Microbiology and Virology and Tbilisi State University, Tbilisi, Georgia, 26-29 June 2017, 105.

[9] Syrym, N.S. and Yespembetov, B.A. (2016) Isolation of Mycobacteriophages from the Environment and Biological Specimens. In: Abstract Book of the III International Scientific-Practical Conference "Bacteriophages: Theoretical and Practical Aspects of Application in Medicine, Veterinary and Food Industries", Moscow, 13-15 October 2016.

[10] Yespembetov, B. (2016) Bacteriophages: Theoretical and Practical Aspects of Application in Medicine, Veterinary Medicine and Food Industry. Proceedings of the Symposium on Phage Therapy at Japanese Agency for Marine-Earth Science and Technology (JAMSTEC), 22 October 2016.

[11] Syrym, N. (2016) Development of a Method for Isolation of Bacteriophages against Animal Tuberculosis. Proceedings of the Symposium on Phage Therapy at JAMSTEC, 22 October 2016.

[12] Yespembetov, B.A. (2017) Electron Microscopy of Mycobacteriophages. In: Abstract Book of the 15th Finnish Microbial Pathogenesis Conference at Haartman Institute, University of Helsinki, Finland, 19-23 August 2017.

[13] Syrym, N.S. and Yespembetov, B.A. (2017) Search and Selection of Mycobacteriophages. In: Abstract Book of the 15th Finnish Microbial Pathogenesis Conference at Haartman Institute, University of Helsinki, Finland, 19-23 August 2017.

[14] Manual on the Diagnosis of Tuberculosis of Animals (1999). Astana, 19 p.

[15] Instructions (1999) On Measures for the Prevention and Elimination of Tuberculosis of Animals. Astana, $29 \mathrm{p}$. 